\title{
On a Limiting Distribution of Singular Values of Random Band Matrices
}

\author{
A. Lytova \\ Department of Mathematical and Statistical Sciences, University of Alberta \\ Edmonton, Alberta, Canada, T6G 2G1 \\ E-mail: lytova@ualberta.ca \\ L. Pastur \\ Theoretical Division, B. Verkin Institute for Low Temperature Physics \\ and Engineering, \\ 47 Lenin Ave., Kharkiv 61103, Ukraine \\ E-mail: pastur@ilt.kharkov.ua
}

\begin{abstract}
An equation is obtained for the Stieltjes transform of the normalized distribution of singular values of non-symmetric band random matrices in the limit when the band width and rank of the matrix simultaneously tend to infinity. Conditions under which this limit agrees with the quarter-circle law are found. An interesting particular case of lower triangular random matrices is also considered and certain properties of the corresponding limiting singular value distribution are given.

Key words: random band matrices, triangular matrices, limiting distribution of singular values.

Mathematics Subject Classifcation 2010: 60B10, 60B20 (primary); 15B52, 15A18 (secondary).
\end{abstract}

\section{Introduction: Problem and Main Results}

Given a positive integer $n=2 m+1, m \in \mathbb{N}$ consider the $n \times n$ random matrix

$$
A_{n}=\left\{A_{j k}^{(n)}\right\}_{|j|,|k| \leq m}, \quad A_{j k}^{(n)}=b_{n}^{-1 / 2} v\left((j-k) / b_{n}\right) a_{j k}^{(n)},
$$

where $\left\{a_{j k}^{(n)}\right\}_{|j|,|k| \leq m}$ are real random variables, $\left\{b_{n}\right\}$ is a sequence of positive integers such that

$$
\lim _{n \rightarrow \infty} b_{n}=\infty, \quad \nu:=\lim _{n \rightarrow \infty} \nu_{n} \in[1, \infty], \quad \nu_{n}=n / 2 b_{n},
$$


$v: \mathbb{R} \rightarrow \mathbb{R}$ is a piecewise continuous function of compact support and we denote

$$
\begin{aligned}
& \int_{-\nu}^{\nu} v^{2}(t) d t=w^{2}<\infty, \\
& \max _{t \in \mathbb{R}} v^{2}(t)=K<\infty .
\end{aligned}
$$

In particular, if $v=\chi_{[0,1]}$, the indicator of the interval $[0,1]$, then the matrix elements $\left\{A_{j k}^{(n)}\right\}_{|j|,|k| \leq m}$ are non-vanishing only in the "band" of the width $b_{n}$ under the principal diagonal. If in addition $2 \nu=1$, then $A_{n}$ is a lower triangular matrix asymptotically.

We are interested in the limiting distribution of the squares of singular values of $A_{n}$, i.e., the eigenvalues

$$
0 \leq \lambda_{1}^{(n)} \leq \ldots \leq \lambda_{n}^{(n)}<\infty
$$

of the positive definite random matrix

$$
M_{n}=A_{n} A_{n}^{T} .
$$

To this end we introduce the Normalized Counting Measure $N_{n}$ of (1.5), setting for any interval $\Delta \subset \mathbb{R}$

$$
N_{n}(\Delta)=\operatorname{Card}\left\{l \in[1, n]: \lambda_{l}^{(n)} \in \Delta\right\} / n .
$$

It is convenient to write matrix $M_{n}$ in the form

$$
M_{n}=\sum_{|k| \leq m} \mathbf{y}_{k} \otimes \mathbf{y}_{k}
$$

where

$$
\mathbf{y}_{k}=\left(A_{-m k}^{(n)}, \ldots, A_{m k}^{(n)}\right)^{T}
$$

are the columns of $A_{n}$ (see (1.1)). According to (1.1), each $\mathbf{y}_{k}$ corresponds to the vector

$$
\mathbf{a}_{k}=\left(a_{-m k}^{(n)}, \ldots, a_{m k}^{(n)}\right)^{T} .
$$

We will assume that $\left\{\mathbf{a}_{k}\right\}_{|k| \leq m}$ are jointly independent random vectors, however the components of each vector $\mathbf{a}_{k}$ can be dependent. Here are the corresponding definitions [9, 14].

Definition 1.1 (i). [Isotropic vectors] A random vector $\mathbf{a}=\left(a_{-m}, \ldots, a_{m}\right) \in \mathbb{R}^{n}$ is called isotropic if

$$
\mathbf{E}\left\{a_{j}\right\}=0, \quad \mathbf{E}\left\{a_{j} a_{k}\right\}=\delta_{j k}, \quad|j|,|k| \leq m .
$$


(ii). [Unconditional distribution] The distribution of random vector $\mathbf{a} \in \mathbb{R}^{n}$ is called unconditional if its components $\left(a_{-m}, \ldots, a_{m}\right)$ have the same joint distribution as $\left( \pm a_{-m}, \ldots, \pm a_{m}\right)$ for any choice of signs.

(iii). [Log-concave measure] A measure $\mu$ on $\mathbb{C}^{n}$ is log-concave if for any measurable subsets $A, B$ of $\mathbb{C}^{n}$ and any $\theta \in[0,1]$,

$$
\mu(\theta A+(1-\theta) B) \geq \mu(A)^{\theta} \mu(B)^{(1-\theta)}
$$

whenever $\theta A+(1-\theta) B=\left\{\theta X_{1}+(1-\theta) X_{2}: X_{1} \in A, X_{2} \in B\right\}$ is measurable.

Definition 1.2 [Good vectors] We say that a random vector $\mathbf{a}=\left(a_{-m}^{(n)}, \ldots, a_{m}^{(n)}\right) \in$ $\mathbb{R}^{n}$ is good, if it is an isotropic vector with an unconditional distribution satisfying the moment conditions

$$
m_{2,2}^{(n)}=\mathbf{E}\left\{\left(a_{j}^{(n)}\right)^{2}\left(a_{k}^{(n)}\right)^{2}\right\}=1+o(1), j \neq k, \quad m_{4}^{(n)}=\mathbf{E}\left\{\left(a_{j}^{(n)}\right)^{4}\right\}=O(1)
$$

as $n=2 m+1 \rightarrow \infty$. Note that $m_{2,2}^{(n)}$ and $m_{4}^{(n)}$ do not depend on $j$ and $k$.

A simple example of good vectors are the vectors with i.i.d. $n$-independent components of zero mean and unit variance. An important case is given by the isotropic random vectors with symmetric unconditional and log-concave distributions (see Lemma 2.1 of [9]), the simplest among them are the vectors uniformly distributed over the unit sphere in $\mathbb{R}^{n}$. Now we are ready to formulate our main results.

Theorem 1.3 Let $M_{n}, n=2 m+1, m \in \mathbb{N}$ be the random matrix (1.8) - (1.10), where for every $m\left\{\mathbf{a}_{k}\right\}_{|k| \leq m}$ are jointly independent good vectors (see Definition 1.2) and corresponding vectors $\left\{\mathbf{y}_{k}\right\}_{|k| \leq m}$ are defined in (1.9) and (1.1) - (1.4). Let $N_{n}$ be the Normalized Counting Measure (1.7) of eigenvalues of $M_{n}$.. Then there exists a non-random and non-negative measure $N, N(\mathbb{R})=1$ such that for any interval $\Delta \subset \mathbb{R}$ we have in probability

$$
\lim _{n \rightarrow \infty} N_{n}(\Delta)=N(\Delta)
$$

The limiting measure $N$ is uniquely defined via its Stieltjes transform $f$ (see [1, 15])

$$
f(z)=\int_{0}^{\infty} \frac{N(d \lambda)}{\lambda-z}, \Im z \neq 0
$$

by the formula

$$
\int_{\mathbb{R}} \varphi(\lambda) N(d \lambda)=\lim _{\varepsilon \rightarrow 0^{+}} \frac{1}{\pi} \int_{\mathbb{R}} \varphi(\lambda) \Im f(\lambda+i \varepsilon) d \lambda, \quad \forall \varphi \in C_{0}(\mathbb{R}),
$$

and we have: 
(i) if $\nu<\infty$ in (1.2), then

$$
f(z)=\frac{1}{2 \nu} \int_{-\nu}^{\nu} f(t, z) d t
$$

where $f:[-\nu, \nu] \times \mathbb{C} \backslash[0, \infty) \rightarrow \mathbb{C}$ on the right of the formula is continuous in $t$ for every $z \in \mathbb{C} \backslash[0, \infty)$, is analytic in z for every $|t| \leq \nu$, has the property

$$
\Im f(t, z) \Im z \geq 0, \quad|f(t, z)| \leq|\Im z|^{-1}, \Im z \neq 0,
$$

and is the unique solution of the equation

$$
f(t, z)=-\left(z-\int_{-\nu}^{\nu} \frac{v^{2}(t-\tau) d \tau}{1+\int_{-\nu}^{\nu} v^{2}(\theta-\tau) f(\theta, z) d \theta}\right)^{-1} .
$$

(ii) if $\nu=\infty$ in (1.2), then $f$ of (1.14) is the unique solution of the quadratic equation

$$
z w^{2} f^{2}+z f+1=0
$$

in the class of analytic in $\mathbb{C} \backslash \mathbb{R}$ functions satisfying

$$
\Im f(z) \Im z \geq 0, \quad|f(z)| \leq|\Im z|^{-1}, \Im z \neq 0,
$$

and we have the following formula for the density $\rho_{q c}$ of the limiting measure $N$ :

$$
N(d \lambda)=\rho_{q c}(\lambda) d \lambda, \quad \rho_{q c}(\lambda)=\left(2 \pi w^{2}\right)^{-1} \sqrt{\left(4 w^{2}-\lambda\right) / \lambda} \mathbf{1}_{\left[0,4 w^{2}\right]},
$$

known as the quarter-circle law.

Theorem 1.4 The results of Theorem 1.3 remain valid if $\left\{\mathbf{a}_{k}\right\}_{|k| \leq m}$ are independent isotropic random vectors with independent components having finite absolute moment of the order $2+\varepsilon, \varepsilon>0$,

$$
\sup _{n} \max _{|j|,|k| \leq m} \mathbf{E}\left\{\left|a_{k j}^{(n)}\right|^{2+\varepsilon}\right\}<\infty .
$$

Corollary 1.5 Under conditions of Theorem 1.3 (or Theorem 1.4) with $\nu<\infty$ in (1.2) $N$ is the quarter-circle law if and only if the function $v^{2}:[-2 \nu, 2 \nu] \rightarrow \mathbb{R}_{+}$ is the restriction on the interval $[-2 \nu, 2 \nu]$ of a $2 \nu$-periodic function.

In particular, if all entries $\left\{A_{j k}^{(n)}\right\}_{|j|,|k| \leq m}$ are non-vanishing, then we get the quarter-circle law, and this fact was proved long time ago [12] (see also [14, 15]).

To prove the corollary, we note first that if $\nu<\infty$ and if $v^{2}$ is $2 \nu$-periodic, then (1.18) has $t$-independent solution $f$, satisfying (1.19). If $v^{2}$ does not possess this property, then the function

$$
u(t) \equiv \int_{-\nu}^{\nu} v^{2}(t-\tau) d \tau
$$


cannot be a constant on the interval $(-\nu, \nu)$. Hence, expanding the solution $f$ of (1.18) in the inverse powers of $z$ :

$$
f(z)=-\frac{1}{z}-\frac{a_{1}}{z^{2}}-\frac{a_{2}}{z^{3}}+O\left(z^{-4}\right), \quad a_{1}=\frac{1}{2 \nu} \int_{-\nu}^{\nu} u(t) d t, \quad a_{2}=\frac{1}{2 \nu} \int_{-\nu}^{\nu} u^{2}(t) d t,
$$

and then applying the Schwarz inequality, we get the strict inequality

$$
a_{1}^{2}<a_{2} / 2,
$$

if $u$ is not identically constant. On the other hand, we have for $f_{q c}$ of (1.21)

$$
f_{q c}(z)=\frac{1}{2 w^{2}}\left(-1+\sqrt{\left(1-4 w^{2} / z\right)}\right)=-\frac{1}{z}-\frac{w^{2}}{z^{2}}-\frac{2 w^{4}}{z^{3}}+O\left(z^{-4}\right), z \rightarrow \infty
$$

so that $a_{1}^{2}=a_{2} / 2$. Therefore, in the considered case of a non-periodic $v^{2}$, the limiting Normalized Counting Measure $N$ of (1.13) cannot be the quarter-circle law.

Note that the results of Theorem 1.3 and Corollary 1.5 agree with those obtained in [4] and [13] for the Wigner band matrices. Indeed, according to [4] and [13] the Stieltjes transform of the Wigner matrices (i.e., matrices with independent, modulo symmetry conditions, entries satisfying an analog of (1.22) is given by the same formula (1.16), where now $f(t, z)$ solves uniquely the equation (cf. (1.18))

$$
f(t, z)=-\left(z+\int_{-\nu}^{\nu} v^{2}(t-\tau) f(z, \tau) d \tau\right)^{-1}
$$

in the same class of functions defined by (1.17)).

Another corollary of Theorem 1.3 yields the liming distribution of singular values of lower triangular random matrices.

Theorem 1.6 Consider the case of Theorem 1.3, where $v=\chi_{[0,1]}$ is the indicator of the interval $[0,1]$ and $2 \nu=1$, so that the matrices $A_{n}$ are lower triangular. Then:

(i) the Stieltjes transform $f$ 1.14) of the limiting Normalized Counting Measure (1.13) of eigenvalues of $M_{n}$ (1.6) solves uniquely the equation

$$
(1+f(z)) \ln (1+f(z))=-z^{-1},
$$

in the class of functions analytic in $\mathbb{C} \backslash[0, \infty)$ and satisfying (1.20);

(ii)

$$
\operatorname{supp} N=[0, e]
$$


(iii) the measure $N$ of (1.13) is absolutely continuous and its density $\rho$ has the following asymptotics at the endpoints of its support $[0, e]$ :

$$
\begin{aligned}
\rho(\lambda) & =\frac{1}{\lambda(\ln \lambda)^{2}}(1+o(1)), \lambda \downarrow 0, \\
\rho(\lambda) & =\text { const } \cdot(e-\lambda)^{1 / 2}(1+o(1)), \lambda \uparrow e .
\end{aligned}
$$

(iv) moments of $N$, i.e., $\mu_{k}:=\lim _{n \rightarrow \infty} n^{-1} \operatorname{Tr} M_{n}^{k}$ are

$$
\mu_{k}=\frac{k^{k}}{(k+1) !}
$$

Remarks. (1). The lower edge $\lambda=0$ of the support is a hard edge in the random matrix terminology. The typical (or standard) soft edge asymptotic of the density of the limiting Normalized Counting Measure near the hard edge is $\rho(\lambda)=$ const $\cdot \lambda^{-1 / 2}, \lambda \downarrow 0^{+}$[15]. The asymptotics (1.25) for the lower triangular matrices seems the most singular among the known so far. It follows from the results of [6] that for the matrices $M_{n}^{(q)}=\left(A_{n}\right)^{q}\left(\left(A_{n}\right)^{q}\right)^{T}$ the soft edge asymptotic of the corresponding density is $\rho(\lambda)=$ const $\cdot\left(\lambda(\ln 1 / \lambda)^{q+1}\right)^{-1}$.

(2). It is of interest that if we replace the lower triangular matrix $A_{n}$ by $A_{n}+y I_{n}$ where $y>0$ and $I_{n}$ is $n \times n$ unit matrix, than it can be shown that the support of the corresponding limiting distribution is $\left[a(y), a_{+}(y)\right]$, for any $y>0, a_{-}(y)>0, a_{+}(y)<\infty$, but $a_{-}(y) \rightarrow 0^{+}, a_{+}(y) \rightarrow e$ as $y \rightarrow 0$ and the both edges of support are soft.

(3). Formula (1.26) was found in [6] by combining the operator and the free probability methods. Our proof is based on the random matrix theory.

To conclude the section we note that the results of Theorem 1.3 for $\nu<\infty$, in particular those for the triangular random matrices generalize in part various results of works [5, 6, 8, 10] obtained for matrices with independent entries by various methods. In Section 3 we outline the proof of Theorem 1.4 treating the case of independent entries under condition (1.22), applicable for both finite and infinite $\nu$ and based on the scheme developed in [15] to find the limiting eigenvalue distribution of a wide variety of random matrices.

\section{Proof of Theorem 1.3}

Recall that if $m$ is a non-negative measure of unit mass and

$$
s(z)=\int_{-\infty}^{\infty} \frac{m(d \lambda)}{\lambda-z}, \Im z \neq 0,
$$


is its Stieltjes transform, then this correspondence is one-to-one, provided that

$$
\Im s(z) \Im z>0, \lim _{n \rightarrow \infty} \eta|s(i \eta)|=1 .
$$

Moreover, the correspondence is continuous if we use the uniform convergence of analytic functions on a compact set of $\mathbb{C} \backslash \mathbb{R}$ for Stieltjes transforms and the weak convergence of probability measures (see e.g. [1, 15]).

Let

$$
g_{n}(z)=\int_{-\infty}^{\infty} \frac{N_{n}(d \lambda)}{\lambda-z}
$$

be the Stieltjes transform of $N_{n}$ of (1.7).

By using the representation (1.8) and repeating almost literally the proof of Theorem 19.1.6 of [15], we obtain the bounds

$$
\mathbf{P}\left\{\left|N_{n}(\Delta)-\mathbf{E}\left\{N_{n}(\Delta)\right\}\right|>\varepsilon\right\} \leq C(\varepsilon) / n,
$$

for any $\Delta \subset \mathbb{R}$, where $C(\varepsilon)$ is independent of $n$ and is finite if $\varepsilon>0$. The bound, the above one-to-one correspondence between the measures and their Stieltjes transforms and the analyticity of $g_{n}$ of $(2.3)$ in $\mathbb{C} \backslash \mathbb{R}$ reduce the proof of the theorem to that of the limiting relation

$$
\lim _{n \rightarrow \infty} \mathbf{E}\left\{g_{n}(z)\right\}=f(z)
$$

uniformly on the set

$$
C_{K_{0}}=\left\{z: \Re z=0,|\Im z| \geq K_{0}>0\right\}
$$

where $K_{0}$ is large enough (see (2.35) and (2.51)).

It follows from (1.7) and the spectral theorem for real symmetric matrices that

$$
g_{n}(z)=\frac{1}{n} \operatorname{Tr} G(z)=\frac{1}{n} \sum_{|j| \leq m} G_{j j}(z),
$$

where

$$
G(z)=\left(M_{n}-z\right)^{-1}
$$

is the resolvent of $M_{n}$. We have

$$
\left|G_{j j}\right| \leq|\Im z|^{-1}, \sum_{k}\left|G_{j k}\right|^{2} \leq|\Im z|^{-2} .
$$

Here and in what follows we use the notation

$$
\sum_{j}=\sum_{|j| \leq m} .
$$


We have by the resolvent identity

$$
G_{j j}=-\frac{1}{z}+\frac{1}{z} \sum_{k}\left(\mathbf{y}_{k} \otimes \mathbf{y}_{k} G\right)_{j j} .
$$

Let us introduce the matrix

$$
M_{n}^{k}=\sum_{l \neq k} \mathbf{y}_{l} \otimes \mathbf{y}_{l}
$$

and its resolvent

$$
G^{k}(z)=\left(M_{n}^{k}-z I_{n}\right)^{-1}, \quad \Im z \neq 0 .
$$

It follows from the rank-one perturbation formula

$$
G-G^{k}=-\frac{G^{k} \mathbf{y}_{k} \otimes \mathbf{y}_{k} G^{k}}{1+\left(G^{k} \mathbf{y}_{k}, \mathbf{y}_{k}\right)}
$$

that

$$
\left(\mathbf{y}_{k} \otimes \mathbf{y}_{k} G\right)_{j j}=\frac{\left(G^{k} \mathbf{y}_{k}\right)_{j} \mathbf{y}_{k j}}{1+\left(G^{k} \mathbf{y}_{k}, \mathbf{y}_{k}\right)} \equiv \frac{B_{k n}}{A_{k n}}
$$

and we obtain from (2.9)

$$
\mathbf{E}\left\{G_{j j}\right\}=-\frac{1}{z}+\frac{1}{z} \sum_{k} \mathbf{E}\left\{\frac{B_{k n}}{A_{k n}}\right\} .
$$

The moment conditions (1.11) and the fact that $G^{k}$ does not depend on $\mathbf{y}_{k}$ allow us to write

$$
\mathbf{E}_{k}\left\{A_{k n}\right\}=1+b_{n}^{-1} \sum_{p} v_{p k}^{2} G_{p p}^{k}, \quad \mathbf{E}_{k}\left\{B_{k n}\right\}=b_{n}^{-1} v_{j k}^{2} G_{j j}^{k},
$$

where

$$
v_{j k}=v\left((j-k) / b_{n}\right)
$$

and $\mathbf{E}_{k}$ denotes the expectation with respect to $\mathbf{y}_{k}$. It follows from (2.15) and the identity

$$
\frac{1}{A}=\frac{1}{\mathbf{E}\{A\}}-\frac{1}{\mathbf{E}\{A\}} \frac{A^{\circ}}{A}, \quad A^{\circ}=A-\mathbf{E}\{A\},
$$

that

$$
\mathbf{E}\left\{G_{j j}\right\}=-\frac{1}{z}+\frac{1}{z b_{n}} \sum_{k} \frac{v_{j k}^{2} \mathbf{E}\left\{G_{j j}^{k}\right\}}{1+b_{n}^{-1} \sum_{p} v_{p k}^{2} \mathbf{E}\left\{G_{p p}^{k}\right\}}+r_{n}
$$


where

$$
r_{n}=-\frac{1}{z} \sum_{k} \frac{1}{\mathbf{E}\left\{A_{k n}\right\}} \mathbf{E}\left\{\frac{\left(A_{k n}\right)^{\circ} B_{k n}}{A_{k n}}\right\}
$$

Let us show that

$$
r_{n}=o(1), \quad n \rightarrow \infty .
$$

By the spectral theorem for the real symmetric matrices there exists a nonnegative measure $m^{k}$ such that

$$
\left(G^{k} \mathbf{y}_{k}, \mathbf{y}_{k}\right)=\int_{0}^{\infty} \frac{m^{k}(d \lambda)}{\lambda-z}
$$

and we can write

$$
\Im\left(z\left(G^{k} \mathbf{y}_{k}, \mathbf{y}_{k}\right)\right)=\Im \int_{0}^{\infty} \frac{\lambda m_{k}(d \lambda)}{\lambda-z}=\Im z \int_{0}^{\infty} \frac{\lambda m_{k}(d \lambda)}{|\lambda-z|^{2}} .
$$

Thus $\Im z \Im\left(z\left(G^{k} \mathbf{y}_{k}, \mathbf{y}_{k}\right)\right) \geq 0$ and

$$
\left|A_{k n}\right|^{-1} \leq\left|\frac{z}{\Im z+\Im\left(z\left(G^{k} \mathbf{y}_{k}, \mathbf{y}_{k}\right)\right)}\right| \leq|z||\Im z|^{-1}=1, \quad z \in C_{K_{0}}
$$

implying the bounds

$$
\left|\mathbf{E}_{k}\left\{A_{k n}\right\}\right|^{-1} \leq 1, \quad\left|\mathbf{E}\left\{A_{k n}\right\}\right|^{-1} \leq 1, \quad z \in C_{K_{0}} .
$$

This and the Schwarz inequality allow us to write for $r_{n}$ of (2.18):

$$
\left|r_{n}\right| \leq \frac{1}{|\Im z|} \sum_{k} \mathbf{E}\left\{\left|\left(A_{k n}\right)^{\circ}\right|^{2}\right\}^{1 / 2} \mathbf{E}\left\{\left|B_{k n}\right|^{2}\right\}^{1 / 2} .
$$

It follows then from (1.12), (2.8) and the bounds (see (1.4))

$$
\max _{s, k} v_{s k}^{2} \leq K, \quad \max _{k} \frac{1}{b_{n}} \sum_{s} v_{s k}^{2} \leq w^{2}
$$

valid for sufficient large $n$, that

$$
\mathbf{E}\left\{\left|B_{k}\right|^{2}\right\}=\frac{v_{j k}^{2}}{b_{n}^{2}} \sum_{s, t} \mathbf{E}\left\{G_{j s}^{k} \overline{G_{j t}^{k}} v_{s k} v_{t k} \mathbf{E}_{k}\left\{a_{s k}^{(n)} a_{t k}^{(n)} a_{j k}^{(n) 2}\right\}\right\} \leq \frac{C v_{j k}^{2}}{|\Im z|^{2} b_{n}^{2}},
$$

where $C$ is an absolute constant. Now (2.19) follows from (2.22) - (2.24) and (2.44).

Let us show that we can replace $G^{k}$ by $G$ in (2.17) with the error of the order $O\left(b_{n}^{-1}\right)$. Indeed, we have from (2.12)

$$
G_{p s}-G_{p s}^{k}=-\frac{\left(G^{k} \mathbf{y}_{k}\right)_{p}\left(G^{k} \mathbf{y}_{k}\right)_{s}}{A_{k n}} .
$$


Applying (2.25), (2.21) and then (1.11), (2.8) and (2.23), we get

$$
\begin{aligned}
\mathbf{E}_{k}\left\{\left|G_{p p}-G_{p p}^{k}\right|\right\} & \leq \mathbf{E}_{k}\left\{\left|\left(G^{k} \mathbf{y}_{k}\right)_{p}\right|^{2}\right\}=\frac{1}{b_{n}} \sum_{s, t} G_{p s}^{k} \overline{G_{p t}^{k}} v_{s k} v_{t k} \mathbf{E}_{k}\left\{a_{s k}^{(n)} a_{t k}^{(n)}\right\} \\
& =\frac{1}{b_{n}} \sum_{s}\left|G_{p s}^{k}\right|^{2} v_{s k}^{2} \leq \frac{K}{b_{n}|\Im z|^{2}}
\end{aligned}
$$

Now it follows from (2.17), in which $G_{j j}^{k}$ is replaced by $G_{j j}$, (2.19) and (2.26) that

$$
\mathbf{E}\left\{G_{j j}\right\}=-\frac{1}{z}+\frac{1}{z b_{n}} \sum_{k} \frac{v_{j k}^{2} \mathbf{E}\left\{G_{j j}\right\}}{1+b_{n}^{-1} \sum_{p} v_{p k}^{2} \mathbf{E}\left\{G_{p p}\right\}}+r_{n j}, \quad n \rightarrow \infty,
$$

where we denote by $r_{n j}$ any reminder satisfying

$$
\sup _{z \in C_{K_{0}}} \max _{j}\left|r_{n j}\right| \rightarrow 0, \quad n \rightarrow \infty
$$

Hence, we have

$$
\mathbf{E}\left\{G_{j j}\right\}=\left(\frac{1}{b_{n}} \sum_{k} \frac{v_{j k}^{2}}{1+b_{n}^{-1} \sum_{p} v_{p k}^{2} \mathbf{E}\left\{G_{p p}\right\}}-z\right)^{-1}\left(1+r_{n j}\right) .
$$

Using (2.20) ant the fact that $\Im G_{p p}(z) \Im z \geq 0, \Im z \neq 0$, it is easy to show that the denominators in (2.27) do not vanish.

Fix $z$ and $n$ and introduce the piece-wise constant function

$$
f_{n}(t, z)=\left\{\begin{array}{cc}
0, & t \notin\left[(-m-1) / b_{n} ; m / b_{n}\right] \\
\mathbf{E}\left\{G_{j j}(z)\right\}, & t \in\left((j-1) / b_{n} ; j / b_{n}\right], \quad|j| \leq m .
\end{array}\right.
$$

We have from (2.7)

$$
\begin{aligned}
\mathbf{E}\left\{g_{n}(z)\right\} & =\frac{b_{n}}{n} \frac{1}{b_{n}} \sum_{j} f_{n}\left(j / b_{n}, z\right)=\frac{b_{n}}{n} \int_{(-m-1) / b_{n}}^{m / b_{n}} f_{n}(t, z) d t \\
& =\frac{1}{2 \nu_{n}} \int_{-\nu_{n}}^{\nu_{n}} f_{n}(t, z) d t+o(1), \quad n \rightarrow \infty,
\end{aligned}
$$

where $\nu_{n}=b_{n} / m \rightarrow \nu, \quad n \rightarrow \infty$ (see (1.2) $)$. Besides, (2.27) implies

$$
f_{n}\left(j / b_{n}, z\right)=\left(\frac{1}{b_{n}} \sum_{k} \frac{v^{2}\left((j-k) / b_{n}\right)}{1+b_{n}^{-1} \sum_{p} v^{2}\left((p-k) / b_{n}\right) f_{n}\left(p / b_{n}, z\right)}-z\right)^{-1}+r_{n j} .
$$

and taking into account (1.3), we get for any $|t| \leq \nu_{n}$

$$
f_{n}(t, z)=\left(\int_{|\tau| \leq \nu_{n}} \frac{v^{2}(t-\tau) d \tau}{1+\int_{|\theta| \leq \nu_{n}} v^{2}(\theta-\tau) f_{n}(\theta, z) d \theta}-z\right)^{-1}+r_{n}(t, z),
$$


where

$$
\lim _{n \rightarrow \infty} \sup _{z \in \leq C_{K_{0}}} \sup _{|t| \leq \nu_{n}}\left|r_{n}(t, z)\right|=0 .
$$

Note now that (1.18) can be written as $f=T f$ where $T$ is a contracting map for any $z \in C_{K_{0}}$. Indeed, we have for any pair $f_{1}, f_{2}$ satisfying (1.17) and any $z \in C_{K_{0}}$

$$
\begin{aligned}
& \left|\int_{|\tau| \leq \nu} v^{2}(t-\tau)\left(1+\int_{|\theta| \leq \nu} v^{2}(\theta-\tau) f_{1,2}(\theta, z) d \theta\right)^{-1} d \tau-z\right|^{-1} \leq K_{0}^{-1} \\
& \left|1+\int_{|\theta| \leq \nu} v^{2}(\theta-\tau) f_{1,2}(\theta, z) d \theta\right|^{-1} \leq\left(1-w^{2} /|\Im z|\right)^{-1} \leq\left(1-w^{2} / K_{0}\right)^{-1}
\end{aligned}
$$

so that

$$
\sup _{|t| \leq \nu}\left|\left[T f_{1}\right](t, z)-\left[T f_{2}\right](t, z)\right| \leq q \sup _{|t| \leq \nu}\left|f_{1}(t, z)-f_{2}(t, z)\right|
$$

where

$$
q \leq \frac{w^{4}}{\left(K_{0}-w^{2}\right)^{2}}<1 \quad \text { if } \quad|\Im z| \geq K_{0}>2 w^{2} .
$$

Hence, for all $z \in C_{K_{0}}$ there exists a unique solution of (1.18) satisfying (1.17).

Consider first the case $\nu<\infty$. Then it follows from (1.4) that for any uniformly bounded in $t, z, n$ functions $\left\{F_{n}\right\}$ we have

$$
\lim _{n \rightarrow \infty} \sup _{z \in C_{K_{0}}} \sup _{|t| \leq \nu_{n}}\left|\left\{\int_{|\tau| \leq \nu_{n}}-\int_{|\tau| \leq \nu}\right\} v^{2}(t-\tau) F_{n}(\tau, z) d \tau\right|=0 .
$$

This, (1.18), (2.30) - (2.31), and (2.34) lead to

$$
\lim _{n \rightarrow \infty} \sup _{z \in C_{K_{0}}} \sup _{|t| \leq \nu_{n}}\left|f_{n}(t, z)-f(t, z)\right|=0 .
$$

hence,

$$
f(z)=\lim _{n \rightarrow \infty} \mathbf{E}\left\{g_{n}(z)\right\}=\lim _{n \rightarrow \infty} \frac{1}{2 \nu_{n}} \int_{|t| \leq \nu_{n} n} f(t, z) d t=\frac{1}{2 \nu} \int_{|t| \leq \nu} f(t, z) d t .
$$

Consider now the case $\nu=\infty$. In this case the unique solution of (1.19) is $t$-independent function $f$, satisfying (1.21). In addition, we have by (2.32)

$$
\frac{1}{2 \nu_{n}} \int_{|t| \leq \nu_{n}}\left|f_{n}(t, z)-f(z)\right| d t \leq K_{0}^{-2} \frac{1}{2 \nu_{n}} \int_{|t| \leq \nu_{n}}\left(T_{1}^{(n)}+T_{2}^{(n)}+T_{3}^{(n)}\right) d t,
$$


where

$$
\begin{gathered}
T_{1}^{(n)=} \int_{|\tau| \leq \nu_{n}} v^{2}(t-\tau) \mid\left(1+\int_{|\theta| \leq \nu_{n}} v^{2}(\theta-\tau) f_{n}(\theta, z) d \theta\right)^{-1} \\
-\left(1+\int_{|\theta| \leq \nu_{n}} v^{2}(\theta-\tau) f(z) d \theta\right)^{-1} \mid d \tau, \\
T_{2}^{(n)=} \int_{|\tau| \leq \nu_{n}} v^{2}(t-\tau) \mid\left(1+\int_{|\theta| \leq \nu_{n}} v^{2}(\theta-\tau) f(z) d \theta\right)^{-1} \\
\quad-\left(1+\int_{|\theta| \leq \infty} v^{2}(\theta-\tau) f(z) d \theta\right)^{-1} \mid d \tau, \\
T_{3}^{(n)=}\left|1+w^{2} h(z)\right|^{-1} \int_{|\tau| \geq \nu_{n}} v^{2}(t-\tau) d \tau .
\end{gathered}
$$

It follows from (1.19) that

$$
\left|1+w^{2} f(z)\right|^{-1}=|z f(z)| \leq 1, \quad \forall z \in C_{K_{0}},
$$

hence,

$$
\begin{aligned}
\frac{1}{2 \nu_{n}} \int_{|t| \leq \nu_{n}} T_{3}^{(n)} d t & \leq \frac{1}{2 \nu_{n}} \int_{|t| \leq \nu_{n}} d t \int_{|\tau| \geq \nu_{n}} v^{2}(t-\tau) d \tau \\
& =\int_{|y| \geq \nu_{n}} v^{2}(y) d y-\frac{1}{2 \nu_{n}} \int_{|y| \leq \nu_{n}}|y| v^{2}(y) d y,
\end{aligned}
$$

and then (1.2) and (1.3) imply

$$
\lim _{n \rightarrow \infty} \frac{1}{2 \nu_{n}} \int_{|t| \leq \nu_{n}} T_{3}^{(n)} d t=0 .
$$

Furthermore, it follows from (2.33) and (2.35) that

$$
\begin{aligned}
\frac{1}{2 \nu_{n}} \int_{|t| \leq \nu_{n}} T_{2}^{(n)} d t & \leq \frac{2}{\nu_{n}} \int_{|t| \leq \nu_{n}} d t \int_{|\tau| \leq \nu_{n}} v^{2}(t-\tau) \int_{|\theta| \geq \nu_{n}} v^{2}(\theta-\tau) d \theta \\
& =\frac{2}{\nu_{n}} \int_{|t| \leq \nu_{n}} d \tau \int_{-\nu_{n}-\tau}^{\nu_{n}-\tau} v^{2}(y) d y \int_{|\theta| \geq \nu_{n}} v^{2}(\theta-\tau) d \theta \\
& \leq \frac{2 w^{2}}{\nu_{n}} \int_{|\tau| \leq \nu_{n}} d \tau \int_{|\theta| \geq \nu_{n}} v^{2}(\theta-\tau) d \theta
\end{aligned}
$$

This and (2.39) yield

$$
\lim _{n \rightarrow \infty} \frac{1}{2 \nu_{n}} \int_{|t| \leq \nu_{n}} T_{2}^{(n)} d t=0 .
$$


We also have

$$
\begin{aligned}
& \frac{1}{2 \nu_{n}} \int_{|t| \leq \nu_{n}} T_{1}^{(n)} d t \leq \frac{2}{\nu_{n}} \int_{|t| \leq \nu_{n}} d t \int_{|\tau| \leq \nu_{n}} v^{2}(t-\tau) d \tau \\
& \quad \times \int_{|\theta| \leq \nu_{n}} v^{2}(\theta-\tau)\left|f_{n}(\theta, z)-h(z)\right| d \theta \\
& \quad=\frac{2}{\nu_{n}} \int_{|\theta| \leq \nu_{n}}\left|f_{n}(\theta, z)-f(z)\right| d \theta \int_{|\tau| \leq \nu_{n}} v^{2}(\theta-\tau) d \tau \int_{|t| \leq \nu_{n}} v^{2}(t-\tau) d \tau \\
& \quad \leq \frac{2 w^{4}}{\nu_{n}} \int_{|\theta| \leq \nu_{n}}\left|f_{n}(\theta, z)-f(z)\right| d \theta
\end{aligned}
$$

It follows from (2.38) and (2.39) - (2.41) that

$$
\left(1-4 w^{4} K_{0}^{-2}\right) \frac{1}{2 \nu_{n}} \int_{|t| \leq \nu_{n}}\left|f_{n}(t, z)-f(z)\right| d t=o(1), \quad n \rightarrow \infty .
$$

We conclude that if $\nu=\infty$, then $f$ of (2.5) coincides with the solution of (1.19) satisfying (1.17). Thus to finish the proof of Theorem 1.3 it remains to prove

Lemma 2.1 Denote $\mathbf{E}_{k}$ the expectation with respect to $\mathbf{y}_{k}$ and let for any random variable $\xi$

$$
\xi_{k}^{\circ}=\xi-\mathbf{E}_{k}\{\xi\}
$$

be its centered version, then we have under the conditions of Theorem 1.3:

(i)

$$
\mathbf{E}\left\{\left|\left(G^{k} \mathbf{y}_{k}, \mathbf{y}_{k}\right)_{k}^{\circ}\right|^{2}\right\} \leq C(z) \delta_{n}
$$

(ii) there exists $K_{0}>0$ such that $\forall z \in C_{K_{0}}=\left\{z: \Re z=0,|\Im z| \geq K_{0}\right\}$

$$
\begin{aligned}
& \operatorname{Var}\left\{G_{p p}\right\} \leq C(z) \delta_{n}, \\
& \operatorname{Var}\left\{\left(G^{k} \mathbf{y}_{k}, \mathbf{y}_{k}\right)\right\} \leq C(z) \delta_{n} .
\end{aligned}
$$

where

$$
\delta_{n}=o(1), \quad n \rightarrow \infty
$$

does not depend on $p, k, z$, and we denote by $C(z)$ any positive quantity, which depends only on $z$ and is finite for $z \in C_{K_{0}}$ (see (2.6))

Proof. It follows from (1.12) and from unconditionality of the distribution of $\mathbf{a}_{k}$ that

$$
\mathbf{E}\left\{a_{p k}^{(n)} a_{q k}^{(n)} a_{s k}^{(n)} a_{t k}^{(n)}\right\}=m_{2,2}^{(n)}\left(\delta_{p q} \delta_{s t}+\delta_{p s} \delta_{q t}+\delta_{p t} \delta_{q s}\right)+\kappa_{4}^{(n)} \delta_{p q} \delta_{p s} \delta_{p t},
$$


where $\kappa_{4}^{(n)}=m_{4}^{(n)}-3\left(m_{2,2}^{(n)}\right)^{2}=O(1), n \rightarrow \infty$. This and (2.15) yield

$$
\begin{aligned}
& \mathbf{E}_{k}\left\{\left|\left(G^{k} \mathbf{y}_{k}, \mathbf{y}_{k}\right)_{k}^{\circ}\right|^{2}\right\}= \\
& =\frac{1}{b_{n}^{2}} \sum_{p, q, s, t} G_{p q} \overline{G_{s t}} v_{p k} v_{q k} v_{s k} v_{t k} \mathbf{E}_{k}\left\{a_{p k}^{(n)} a_{q k}^{(n)} a_{s k}^{(n)} a_{t k}^{(n)}\right\}-\left|\frac{1}{b_{n}} \sum_{p} v_{p k}^{2} G_{p p}^{k}\right|^{2} \\
& =\left(m_{2,2}^{(n)}-1\right)\left|\frac{1}{b_{n}} \sum_{p} v_{p k}^{2} G_{p p}^{k}\right|^{2}+\frac{2 m_{2,2}^{(n)}}{b_{n}^{2}} \sum_{p, s} v_{s k}^{2} v_{p k}^{2}\left|G_{p s}^{k}\right|^{2}+\frac{\kappa_{4}^{(n)}}{b_{n}^{2}} \sum_{p} v_{p k}^{4}\left|G_{p p}^{k}\right|^{4} .
\end{aligned}
$$

By (2.8) and (2.23) we have

$$
\begin{aligned}
& \left|\frac{1}{b_{n}} \sum_{p} v_{p k}^{2} G_{p p}^{k}\right| \leq \frac{K}{|\Im z|}, \\
& \frac{1}{b_{n}^{2}} \sum_{p, s} v_{s k}^{2} v_{p k}^{2}\left|G_{p s}^{k}\right|^{2} \leq \frac{K}{b_{n}^{2}} \sum_{p} v_{p k}^{2} \sum_{s}\left|G_{p s}^{k}\right|^{2} \leq \frac{K^{2}}{b_{n}|\Im z|^{2}} .
\end{aligned}
$$

This, (1.12) and (2.47) lead to (2.42).

Let us prove (2.43). We have (cf. (2.14))

$$
\begin{aligned}
\mathbf{E}\left\{G_{j j} \overline{G_{j j}^{\circ}}\right\}=\frac{1}{z} \sum_{k} \mathbf{E}\left\{\frac{B_{k n}}{A_{k n}} \overline{G_{j j}^{\circ}}\right\}= & \frac{1}{z} \sum_{k} \mathbf{E}\left\{\mathbf{E}_{k}\left\{\frac{B_{k n}}{A_{k n}}\right\} \overline{G_{j j}^{k \circ}}\right\} \\
& +\frac{1}{z} \sum_{k} \mathbf{E}\left\{\frac{B_{k n}}{A_{k n}} \overline{\left(G_{j j}-G_{j j}^{k}\right)^{\circ}}\right\}=: \mathcal{T}_{1}+\mathcal{T}_{2} .
\end{aligned}
$$

It follows from the Schwarz inequality, (2.21), (2.24) and (2.26):

$$
\left|\mathcal{T}_{2}\right| \leq \sum_{k} \mathbf{E}\left\{\left|B_{k}\right|^{2}\right\}^{1 / 2} \mathbf{E}\left\{\left|\left(G_{p p}-G_{p p}^{k}\right)^{\circ}\right|^{2}\right\}^{1 / 2} \leq C(z) b_{n}^{-1 / 2}
$$

Consider now $\mathcal{T}_{1}$ of (2.49). We have by (2.16)

$$
\mathbf{E}_{k}\left\{\frac{B_{k n}}{A_{k n}}\right\}=\frac{\mathbf{E}_{k}\left\{B_{k n}\right\}}{\mathbf{E}_{k}\left\{A_{k n}\right\}}-\frac{1}{\mathbf{E}_{k}\left\{A_{k n}\right\}} \mathbf{E}_{k}\left\{\frac{\left(A_{k n}\right)_{k}^{\circ} B_{k n}}{A_{k n}}\right\},
$$

and by the Schwarz inequality, (2.21), (2.24), and (2.42)

$$
\mathbf{E}_{k}\left\{A_{k n}{ }^{-1}\left(A_{k n}\right)_{k}^{\circ} B_{k n}\right\} \leq C(z) b_{n}^{-1}\left|v_{j k}\right| \delta_{n}^{1 / 2} .
$$

This and (2.15) yield

$$
\mathcal{T}_{1}=\frac{1}{z b_{n}} \sum_{k} v_{j k}^{2} \mathbf{E}\left\{\frac{1}{\mathbf{E}_{k}\left\{A_{k n}\right\}} G_{j j}^{k} \overline{G_{j j}^{k \circ}}\right\}+o(1), \quad n \rightarrow \infty .
$$


Applying again (2.16) and then (2.15), we get

$$
\begin{array}{r}
\mathbf{E}\left\{\frac{G_{j j}^{k} \overline{G_{j j}^{k \circ}}}{\mathbf{E}_{k}\left\{A_{k n}\right\}}\right\}=\frac{1}{\mathbf{E}\left\{A_{k n}\right\}} \mathbf{E}\left\{\left|G_{j j}^{k \circ}\right|^{2}\right\}-\frac{1}{\mathbf{E}\left\{A_{k n}\right\}} \mathbf{E}\left\{\frac{\left(\mathbf{E}_{k}\left\{A_{k n}\right\}\right)^{\circ}}{\mathbf{E}_{k}\left\{A_{k n}\right\}} G_{j j}^{k} \overline{G_{j j}^{k \circ}}\right\} \\
=\frac{1}{\mathbf{E}\left\{A_{k n}\right\}} \mathbf{E}\left\{\left|G_{j j}^{k \circ}\right|^{2}\right\}-\frac{1}{\mathbf{E}\left\{A_{k n}\right\}} \frac{1}{b_{n}} \sum_{p} v_{p k}^{2} \mathbf{E}\left\{\frac{G_{p p}^{k \circ}}{\mathbf{E}_{k}\left\{A_{k n}\right\}} G_{j j}^{k} \overline{G_{j j}^{k \circ}}\right\} .
\end{array}
$$

Note also that in view of (2.26) we can replace $G^{k}$ with $G$ with the error term of the order $O\left(b_{n}^{-1}\right)$, hence

$$
\begin{aligned}
\mathcal{T}_{1}= & \frac{1}{z b_{n}} \sum_{k} v_{j k}^{2} \frac{1}{\mathbf{E}\left\{A_{k n}\right\}} \cdot \mathbf{E}\left\{\left|G_{j j}^{\circ}\right|^{2}\right\} \\
& -\frac{1}{z b_{n}^{2}} \sum_{k} v_{j k}^{2} \frac{1}{\mathbf{E}\left\{A_{k n}\right\}} \sum_{p} v_{p k}^{2} \mathbf{E}\left\{\frac{G_{j j}^{k}}{\mathbf{E}_{k}\left\{A_{k n}\right\}} G_{p p}^{\circ} \overline{G_{j j}^{\circ}}\right\}+o(1), \quad n \rightarrow \infty
\end{aligned}
$$

and by the Schwarz inequality, (2.21), and (2.23)

$$
\left|\mathcal{T}_{1}\right| \leq \frac{K}{|\Im z|} \operatorname{Var}\left\{G_{j j}\right\}+\frac{K^{2}}{|\Im z|^{2}} \operatorname{Var}\left\{G_{j j}\right\}^{1 / 2} \max _{|p| \leq m} \operatorname{Var}\left\{G_{p p}\right\}^{1 / 2}+o(1), \quad n \rightarrow \infty .
$$

This and (2.48) - (2.49) yield for $V_{j}:=\operatorname{Var}\left\{G_{j j}\right\},|j| \leq m, z \in C_{K_{0}}$ :

$$
V_{j} \leq \frac{K}{K_{0}} V_{j}+\frac{K^{2}}{K_{0}^{2}} V_{j}^{1 / 2} \max _{|p| \leq m} V_{p}^{1 / 2}+o(1), \quad n \rightarrow \infty
$$

Choosing here $K_{0}$ such that

$$
K / K_{0}+K^{2} / K_{0}^{2}<1
$$

we obtain that

$$
\max _{|p| \leq m} V_{p}=o(1), n \rightarrow \infty
$$

i.e., (2.43).

It remains to note that we have by (2.15)

$$
\left(G^{k} \mathbf{y}_{k}, \mathbf{y}_{k}\right)^{\circ}=\left(G^{k} \mathbf{y}_{k}, \mathbf{y}_{k}\right)_{k}^{\circ}+b_{n}^{-1} \sum_{p} v_{p k}^{2} G_{p p}^{k \circ}
$$

This together with (2.42) - (2.43) lead to (2.44) and complete the proof of the lemma. 


\section{Proof of Theorem 1.4}

The proof of Theorem 1.4 can be obtained by following the scheme worked out in [11] (see also [15] and references therein) and applicable to a wide variety of random matrices with independent entries. Namely, one uses first the martingaletype argument to prove the bound (2.4) and then the so-called interpolation trick to reduce the initial problem to that one of finding the limit (2.5) for the random matrices with Gaussian entries with the same first and second moments. Since these two steps are rather standard, we will explain below just the derivation of the limiting equations (1.16) - (1.17) for i.i.d. Gaussian entries $\left\{a_{j k}\right\}_{|j|,|k| \leq m}$ satisfying (1.11). Note also that by using a standard truncation technique condition (1.22) can be replaced with the Lindeberg type condition for the second moments (see [11, 15]).

Accordingly, consider a random matrix $A_{n}$ (1.1) - (1.4), where $\left\{a_{j k}\right\}_{|j|,|k| \leq m}$ are jointly independent standard Gaussian random variables of zero mean and unit variance. We will use

Proposition 3.1 Let $\xi=\left\{\xi_{l}\right\}_{l=1}^{p}$ be independent Gaussian random variables of zero mean, and $\Phi: \mathbb{R}^{p} \rightarrow \mathbb{C}$ be a differentiable function with polynomially bounded partial derivatives $\Phi_{l}^{\prime}, l=1, \ldots, p$. Then we have

$$
\mathbf{E}\left\{\xi_{l} \Phi(\xi)\right\}=\mathbf{E}\left\{\xi_{l}^{2}\right\} \mathbf{E}\left\{\Phi_{l}^{\prime}(\xi)\right\}, l=1, \ldots, p,
$$

and

$$
\operatorname{Var}\{\Phi(\xi)\} \leq \sum_{l=1}^{p} \mathbf{E}\left\{\xi_{l}^{2}\right\} \mathbf{E}\left\{\left|\Phi_{l}^{\prime}(\xi)\right|^{2}\right\}
$$

The first formula is a version of the integration by parts. The second is a version of the Poincaré inequality (see e.g. [15]).

We have by the resolvent identity and (3.1)

$$
\mathbf{E}\left\{G_{j j}(z)\right\}=-\frac{1}{z}+\frac{1}{z b_{n}} \sum_{k} v_{j k}^{2} \mathbf{E}\left\{D_{j k}\left(G(z) A_{n}\right)_{j k}\right\},
$$

where $D_{j k}=\partial / \partial A_{j k}^{(n)}$. It can be shown that

$$
D_{j k}\left(G A_{n}\right)_{j k}=-z \widetilde{G}_{k k} G_{j j}-\left(G A_{n}\right)_{j k}^{2}
$$

(see, e.g., [11]), where $\widetilde{G}=\left(\widetilde{M}-z I_{n}\right)^{-1}, \widetilde{M}=A_{n}^{T} A_{n}$. Hence,

$$
\begin{aligned}
\mathbf{E}\left\{G_{j j}(z)\right\} & =-\frac{1}{z}-\frac{1}{b_{n}} \sum_{k} v_{j k}^{2} \mathbf{E}\left\{\widetilde{G}_{\alpha \alpha}(z) G_{j j}(z)\right\}+r_{n}(z), \\
r_{n}(z) & =-\frac{1}{b_{n}} \sum_{k} v_{j k}^{2} \mathbf{E}\left\{\left(G A_{n}\right)_{j k}^{2}\right\} .
\end{aligned}
$$


Since

$$
\sum_{k}\left|\left(G A_{n}\right)_{j k}^{2}\right|=\left(G M_{n} \bar{G}\right)_{j j}=\left(\left(I_{n}+z G\right) \bar{G}\right)_{j j}
$$

then

$$
r_{n}=O\left(b_{n}^{-1}\right), \quad n \rightarrow \infty .
$$

We also have by (3.2) - (3.3)

$$
\begin{aligned}
\operatorname{Var}\left\{G_{j j}(z)\right\} & \leq \sum_{l, k} \mathbf{E}\left\{\left|\partial G_{j j} / \partial a_{l k}\right|^{2}\right\} \\
& \leq \frac{4}{b_{n}} \sum_{l, k} v_{l k}^{2} \mathbf{E}\left\{\left|\left(G A_{n}\right)_{j l} G_{j k}\right|^{2}\right\} \leq C(z) b_{n}^{-1}
\end{aligned}
$$

Now it follows from (3.4) - (3.6) that

$$
\mathbf{E}\left\{G_{j j}(z)\right\}=-\frac{1}{z}-\frac{1}{b_{n}} \sum_{k} v_{j k}^{2} \mathbf{E}\left\{\widetilde{G}_{k k}(z)\right\} \mathbf{E}\left\{G_{j j}(z)\right\}+O\left(b_{n}^{-1 / 2}\right),
$$

as $n \rightarrow \infty$. Similarly,

$$
\mathbf{E}\left\{\widetilde{G}_{k k}(z)\right\}=-\frac{1}{z}-\frac{1}{b_{n}} \sum_{p} v_{p k}^{2} \mathbf{E}\left\{G_{p p}(z)\right\} \mathbf{E}\left\{\widetilde{G}_{k k}(z)\right\}+O\left(b_{n}^{-1 / 2}\right),
$$

as $n \rightarrow \infty$. Solving system (3.7) - (3.8), we get (2.27) and then it suffices to use the same argument as that in the proof of Theorem 1.3 to finish the proof of Theorem 1.4.

\section{Triangular matrices}

In this section we prove Theorem 1.6. It follows from (1.18) that if $v(t)=\chi_{[0 ; 1]}(t)$ and $\nu=1 / 2$, then

$$
f(t, z)=-\left(z-\int_{-1 / 2}^{t}\left(1+\int_{\tau}^{1 / 2} f(\theta, z) d \theta\right)^{-1} d \tau\right)^{-1} .
$$

Denote

$$
\varphi(t, z)=\int_{t}^{1 / 2} f(\theta, z) d \theta .
$$

It follows from (4.1) - 4.2) that

$$
\varphi^{\prime \prime}-\varphi^{\prime 2}(1+\varphi)^{-1}=0, \varphi(1 / 2, z)=0, \varphi^{\prime}(1 / 2, z)=z^{-1}
$$


where $\varphi^{\prime}=\partial \varphi / \partial t$. Solving this system, we get for $f(z)=\varphi(-1 / 2, z)$ :

$$
f(z)=e^{c(z)}-1, c(z) e^{c(z)}=-z^{-1}, z \in \mathbb{C} \backslash[0 ; \infty) .
$$

These equations are equivalent to (1.23). Evidently, there is only one solution $c$ analytical in $\mathbb{R} \backslash[0, \infty)$.

Let us prove (1.24). As it was firstly shown in [12] (see also [3, 15]), to find the support of measure $N$, it suffices to consider function $x=x(f), f \in \mathbb{R}$, which is the functional inverse of Stieltjes transform of $N$, and to find set $L \subset \mathbb{R}$ on which $x$ increases monotonically. Then supp $N=\overline{\mathbb{R} \backslash x(L)}$, where $x(L)=\{x(f): f \in L\}$.

It follows from (4.3) that in our case

$$
x(f)=-\frac{1}{(1+f) \ln (1+f)}, \quad f>-1, f \neq 0 .
$$

It is easy to find that $x(f)$ increases on $L=\left[e^{-1}, 0\right) \cup(0, \infty)$. Thus $x(L)=$ $(-\infty, 0) \cup[e, \infty)$ and $\operatorname{supp} N=\overline{\mathbb{R} \backslash x(L)}=[0, e]$.

To prove asymptotic relations (1.25), we first consider

$$
F(x):=f(-x)=\int_{0}^{\infty} \frac{N(d \lambda)}{\lambda+x}, \quad x>0 .
$$

It is easy to find from (1.23) that

$$
F(x)=\frac{1}{x \ln 1 / x}(1+o(1)), \quad x \downarrow 0 .
$$

This and the Tauberain theorem (see [7, Chapter XIII.5) imply

$$
N(\lambda):=N([0, \lambda])=\frac{1}{\ln 1 / \lambda}(1+o(1)), \quad \lambda \downarrow 0 .
$$

Differentiating formally this asymptotic formula we obtain the first formula of (1.25).

To prove this formula rigorously we use (4.3). Denoting $c(\lambda+i 0)=\xi(\lambda)+$ $i \eta(\lambda)$, we obtain from (4.3) and (1.15)

$$
\rho(\lambda)=\frac{\sin ^{2} \eta}{\pi \lambda \eta}, \quad \lambda=\frac{e^{\eta \cot \eta} \sin \eta}{\eta}, \quad \eta \in[0, \pi] .
$$

Since the limit $\lambda \downarrow 0$ corresponds to $\eta=\pi-\sigma, \sigma \downarrow 0$, we have from (4.6) $\ln \lambda=-\pi / \sigma+O(\ln \sigma), \sigma \downarrow 0$ and eventually the first asymptotics of (1.25). The second asymptotics of (1.25) can be obtained similarly taking into account that the limit $\lambda \uparrow e$ corresponds to the limit $\eta \downarrow 0$. 
Let us prove (1.26). To this end we will use the identity

$$
\mu_{k}:=\frac{k^{k}}{(k+1) !}=\frac{1}{2 \pi i k} \int_{|\zeta|=\varepsilon} \frac{e^{k \zeta}}{\zeta^{k+2}} d \zeta, \quad \varepsilon>0, \quad k \geq 1 .
$$

Consider the generating function

$$
h(z)=-\sum_{l=0}^{\infty} \frac{\mu_{l}}{z^{l+1}}
$$

which is well defined if $z$ is sufficiently large. We have then the integral representation

$$
z h(z)=-1+\frac{1}{2 \pi i} \int_{|\zeta|=\varepsilon} \log \left(1-\frac{e^{\zeta}}{\zeta z}\right) \frac{d \zeta}{\zeta^{2}}
$$

or

$$
(z h(z))^{\prime}=\frac{1}{2 \pi i z} \int_{|\zeta|=\varepsilon} \frac{d \zeta}{\zeta^{2}\left(e^{-\zeta} \zeta z-1\right)} .
$$

The both formulas are valid for $|z|>e^{\varepsilon} \varepsilon^{-1}$, where the integrands are analytic in $\zeta$ just because the series for the integrands are convergent. It is easy to find that the function $u_{z}(\zeta)=e^{-\zeta} \zeta z-1$ has a simple zero in $\zeta(z)=z^{-1}(1+o(1)), z \rightarrow \infty$, i.e., inside the contour $|\zeta|=\varepsilon$ if $\varepsilon$ does not depend on $z$. Thus the integral on the left of (4.7) is equal to $z^{-1}$ times the residue of $u_{z}$ at $\zeta(z)$ (i.e., $(\zeta(z)(1-\zeta(z)))^{-1}$ ) plus the integral over a sufficiently "small" contour, say $|\zeta|=|2 z|^{-1}$. Since $u_{z}$

has no zeros inside this contour, the corresponding integral is just $u_{z}^{\prime}(0)=-z$. Putting everything together, we obtain

$$
(z h(z))^{\prime}=(z \zeta(z)(1-\zeta(z)))^{-1}-1, z \zeta(z) e^{-\zeta(z)}=1 .
$$

On the other hand, it follows from (4.3) that $(z f(z))^{\prime}=-(z c(z)(1+c(z)))^{-1}-1$. Thus, setting $\zeta=-c$ we obtain that $h$ coincides with $f$, i.e., assertion (iv) of the theorem.

\section{References}

[1] N. I. Akhiezer and I. M. Glazman, Theory of Linear Operators in Hilbert Space. Dover, New York, 1993.

[2] A. Boutet de Monvel, A. Khorunzhy, and V. Vasilchuk, Limiting eigenvalue distribution of random matrices with correlated entries. - Markov Process. Related Fields, 24 (1996), 607-636.

[3] Z. D. Bai and J. W. Silverstein, Spectral Analysis of Large Dimensional Random Matrices. Springer, New York, 2010. 
[4] G. Casati and V. Girko, Wigner's semicircle law for band random matrices. - Random Operators and Stochastic Equations, vol. 11 (1993), 15-22.

[5] B. Collins, P. Gawron, A. E. Litvak, and K. Zyczkowski, Numerical range for random matrices. - J. Math. Anal. Appl. 4181 (2014), 516-533.

[6] K. Dykema and U. Haagerup, DT-operators and decomposability of Voiculescu's circular operator. - Amer. J. Math. 1261 (2004), 121 - 189.

[7] W. Feller, An introduction to probability theory and its applications. Vol. 2. John Wiley \& Sons, 2008.

[8] V. L. Girko, Theory of stochastic canonical equations. Vol. I, Kluwer Academic Publishers, Dordrecht, 2001.

[9] O. Guedon, A. Lytova, A. Pajor, and L. Pastur, The Central Limit Theorem for linear eigenvalue statistics of the sum of rank one projections on independent vectors. Spectral Theory and Differential Equations. V. A. Marchenko 90th Anniversary Collection (editors: E.Khruslov and L.Pastur, AMS Providence, RI, 2014). available at arXiv:1310.2506

[10] W. Hachem, Ph. Loubaton, and J. Najim, Deterministic Equivalents for Certain Functionals of Large Random Matrices. - Ann. of Appl. Prob. 17 (2007), No. 3

[11] A. Lytova and L. Pastur, Central limit theorem for linear eigenvalue statistics of random matrices with independent entries. - Ann. of Prob. 37 (2009), No. $5,1778-1840$.

[12] V. Marchenko and L. Pastur, The eigenvalue distribution in some ensembles of random matrices. - Math. USSR Sbornik 1 (1967), 457-483.

[13] S. A. Molchanov, L. A. Pastur, and A. M. Khorunzhii, Limiting Eigenvalue Distribution for Band Random Matrices. - Teor. Mat. Fizißka, 90 (1992), No. 2, 163-178.

[14] A. Pajor and L. Pastur, On the limiting empirical measure of eigenvalues of the sum of rank one matrices with log-concave distribution. - Studia Math. 195 (2009), 11-29.

[15] L. Pastur and M. Shcherbina, Eigenvalue Distribution of Large Random Matrices, Math. Surveys and Monographs. Amer. Math. Soc. 171 (2011) 634. 